\title{
TV/Series
}

19 | 2021

Perfectionnisme et séries télévisées. Hommage à Stanley Cavell (1926-2018)

\section{Black Mirror (S03E01) ou de l'imperfectionnisme moral à l'écran. Quelles voies pour une vie bonne et connectée?}

Laurence Allard

\section{(c) OpenEdition}

Journals

Édition électronique

URL : https://journals.openedition.org/tvseries/5440

DOI : $10.4000 /$ tvseries. 5440

ISSN : 2266-0909

Éditeur

GRIC - Groupe de recherche Identités et Cultures

Référence électronique

Laurence Allard, «Black Mirror (S03E01) ou de l'imperfectionnisme moral à l'écran. Quelles voies pour une vie bonne et connectée? », TV/Series [En ligne], 19 | 2021, mis en ligne le 06 mai 2021, consulté le 14 mai 2021. URL : http://journals.openedition.org/tvseries/5440 ; DOl : https://doi.org/10.4000/ tvseries. 5440

Ce document a été généré automatiquement le 14 mai 2021

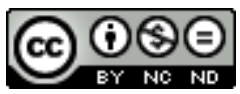

TV/Series est mis à disposition selon les termes de la licence Creative Commons Attribution - Pas d'Utilisation Commerciale - Pas de Modification 4.0 International. 


\section{Black Mirror (S03E01) ou de l'imperfectionnisme moral à l'écran. Quelles voies pour une vie bonne et connectée?}

Laurence Allard

"Les auteurs de SF cauchemardent le monde parce qu'ils savent que le bonheur est possible»

Norbert Merjagnan ${ }^{1}$

\section{Introduction}

1 Cet article voudrait s'attarder sur un épisode bien connu la série britannique Black Mirror, créée Charlie Brooker et diffusée sur Channel 4 (2011-2014), puis sur Netflix (2016-), « Nosedive » (ou « Chute libre »), premier épisode de la troisième saison (2016). Nous nous attacherons en particulier à typifier les formes de vie socio-numériques qui y sont caricaturées suivant une prospective d'imperfectionnisme moral. Cet horizon d'imperfectionnisme moral vient questionner la vision de Stanley Cavell de constituer les relations interpersonnelles comme substrat nécessaire à la possibilité de transformation de notre société 2 . En effet, les épisodes de la série Black Mirror viennent mettre en scène des interactions sociales qui se trouvent médiatisées par des artefacts technologiques telles que les applications de notation sociale pilotées par le nombre de like. Les formes de vie qui se trouvent projetées dans cette série fictionnant les expérimentations les plus extrêmes des dernières innovations technologiques possèdent des effets performatifs puissants que l'expression tendant à devenir commune « c'est comme dans Black Mirror » traduit. L'effet de réel de la projection d'un monde pointée par Cavell ${ }^{3}$ est parfaitement à l'œuvre dans la série. Le monde numérisé contemporain est désormais perçu à travers le miroir noir de cette série, un miroir grossissant, un miroir déformant. Si toute fiction représente un monde possible, Black Mirror est reçu comme proposant un monde prévisible. Le pouvoir transformateur de la 
fiction est ainsi détourné au profit d'un déjà là projeté qui par avance réduit à l'impuissance toute contestation possible. Nous proposerons une voie/voix critique permettant échapper à une passive consommation de la dystopie au profit d'un "contre-faire» et d'une re-fabrication d'un autre monde connecté possible écologiquement soutenable.

\section{Imperfectionnisme moral/perfectionnisme moral : mises en conversations de la notation sociale connectée dans «Chute Libre » et ses réceptions}

2 Á la manière de Cavell qui, au départ de sa démarche, ne veut "pas produire de définition » du perfectionnisme moral ${ }^{4}$ mais en explore le dessin en en éclairant successivement des aspects, nous allons aborder l'épisode «Nosedive» (ou "Chute libre $»)^{5}$ suivant cette perspective en nous focalisant sur certaines séquences souvent discutées lors de projections-débats auxquels j'ai contribué soit dans des festivals de séries ou des ciné-clubs étudiants. En nous appuyons sur une méthodologie explorée par mes soins dans une autre publication ${ }^{6}$, je me baserai également sur les tweets rédigés lors d'un visionnement de "Chute Libre» puisque désormais les épisodes peuvent être téléchargés hors des régulations de la chronologie des médias ${ }^{7}$. Ce choix méthodologique de corpus saisi depuis des réceptions publiques permet d'emblée en dialogue en dialogue nos analyses et ainsi de se mettre au diapason d'une exploration intersubjective de ce que pourrait être une bonne vie connectée.

3 La série Black Mirror s'est fait connaître par ce slogan « la technologie vous drogue, quels en sont ses effets ${ }^{8}$ ? " et met en scène une vision d'imperfectionnisme moral qui nous questionne a contrario sur nos jugements moraux à travers le spectacle d'interactions médiatisés par des applications de réseaux sociaux (voir «Haine Virtuelle », S03E06) ou autres artefacts techno-prothésistes de mémorisation («Retour sur image », S01E03) ou de localisation (« Archange », S04E02).

\subsection{La méthode de la " technologie fictive » : startup, SF, même combat?}

4 La méthode scénaristique de Black Mirror consiste, comme l'ont explicité les co-auteurs de la série, Charlie Brooker et Annabel Jones', à fabuler autour des dernières innovations technologiques et à s'inspirer d'une veille intensive tout autant du côté des concepteurs (startups, états) que des usages émergents. La « technologie fictive » est de fait un genre en soi dans la culture des entreprises de la high-tech présentant à des fins de financement sous la forme de keynote et autres pitchs des services prototypés mais pas ou peu encore développés. La performativité du discours d'escorte dans la médiation sociale d'une nouvelle technologie et les différentes traductions qu'un objet technique connait au cours de ce processus qui va du financement à l'usage ${ }^{10}$ est observable en de nombreuses circonstances. Il y a d'ailleurs certains romanciers cyberpunks qui ont intégré des sociétés technologiques et se trouvent nommés chief futurist, tel Neal Stephenson embauché depuis 2014 chez Leap Motion et y développant des applications de réalité augmentée. Et c'est une évidence que de rappeler que le genre littéraire qui a consacré l'invention technologique comme matière à fiction est 
évidemment la science-fiction qui joue aujourd'hui, suivant notre hypothèse, le rôle de support culturel à la critique sociale qu'a pu jouer le polar français en France et aux USA $^{11}$. Le succès de l'œuvre d'Alain Damasio et le poids de sa parole, à travers les débats et autres manifestations auquel l'auteur participe où il dénonce une société connectée dominée par le capitalisme de plateforme, en est emblématique ${ }^{12}$.

Dans la réception publique de Black Mirror, l'inscription des co-scénaristes dans le sillage du genre science-fiction est rarement mise en doute. Dans les débats auxquels j'ai pu participer l'expression de «dystopie » est le plus souvent citée ${ }^{13}$ et c'est désormais au filtre de ce miroir noircissant les conduites interindividuelles de nos sociétés numérisées que peut s'effectuer l'appréhension des formes de vie contemporaines.

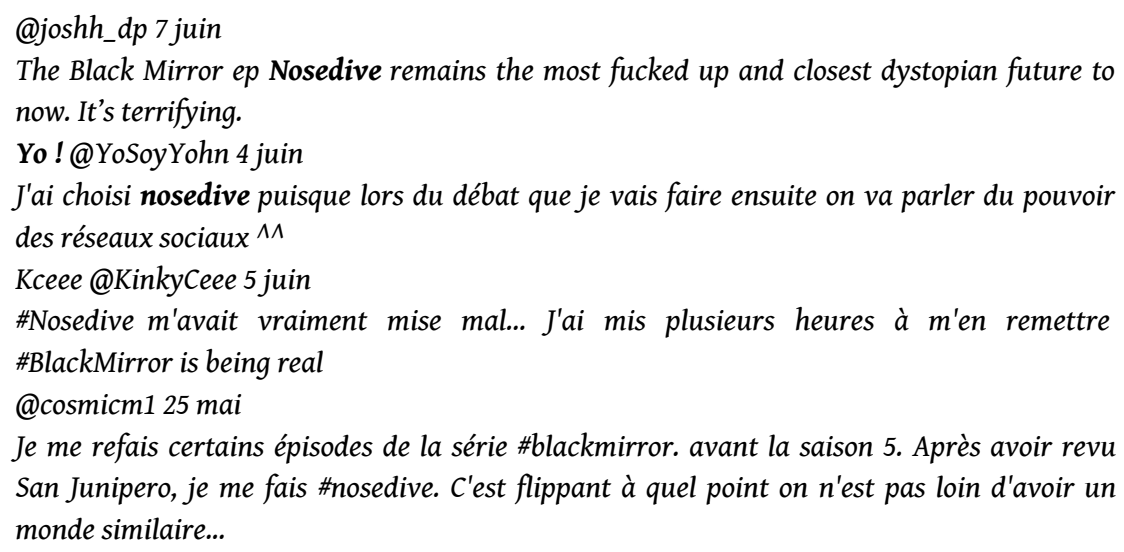

\subsection{La mise en conversation : du jugement moral à l'enquête collective}

Dans un premier temps, cette projection d'un monde qui réalise le pouvoir de la fiction et son heuristique en mettant en forme les fils mêlés de la vie ordinaire ${ }^{14}$ permet d'éprouver l'imperfectionnisme moral et faire se réaliser dans l'intersubjectivité spectactorielle une sorte d'enquête sur la société numérique et ses problèmes menée par un public au sens de John Dewey ${ }^{15}$. Dans un second temps, nous déplierons les limites, suivant la perspective transformatrice du perfectionnisme moral cavellien, de cette posture spectatorielle d'une critique sociale plateformisée par un acteur devenu majeur dans la production numérique des fictions et des imaginaires, en l'occurrence Netflix qui, depuis la saison 3, a succédé à Endemol (grand pourvoyeur par ailleurs d'émissions de la télé-réalité).

$7 \mathrm{Au}$ sein du corpus de tweets rédigés pendant les visionnements de "Chute libre $»^{16}$, il apparait un sous-corpus exemplaire comprenant un ensemble de jugements moraux adressés à cet univers filmé, qui présente, sous une esthétique rose bonbon, un monde de relations sociales indexées à une notation impitoyable des interactions.

@SravaniWrites 2 juin

What a perfect writerly aesthetic. I feel like Lacie from \#BlackMirror Nosedive.

\#AmWriting

8 De même que l'on peut parler d'un « double agir communicationnel » quand il s'agit de lire/dire et voir une fiction à travers les jeux de langage du livetweeting ${ }^{17}$, de même émettre un jugement moral sur des interactions dont la validation n'est plus intersubjective mais calculée nous oblige à faire l'analyse d'un double mouvement 
entre imperfectionnisme et perfectionnisme moral. Le langage ordinaire est ainsi la matière même de transactions sociales qu'il va s'agir non seulement d'accomplir et de valider mais également de noter et ce à la fois au sein et à propos de l'épisode.

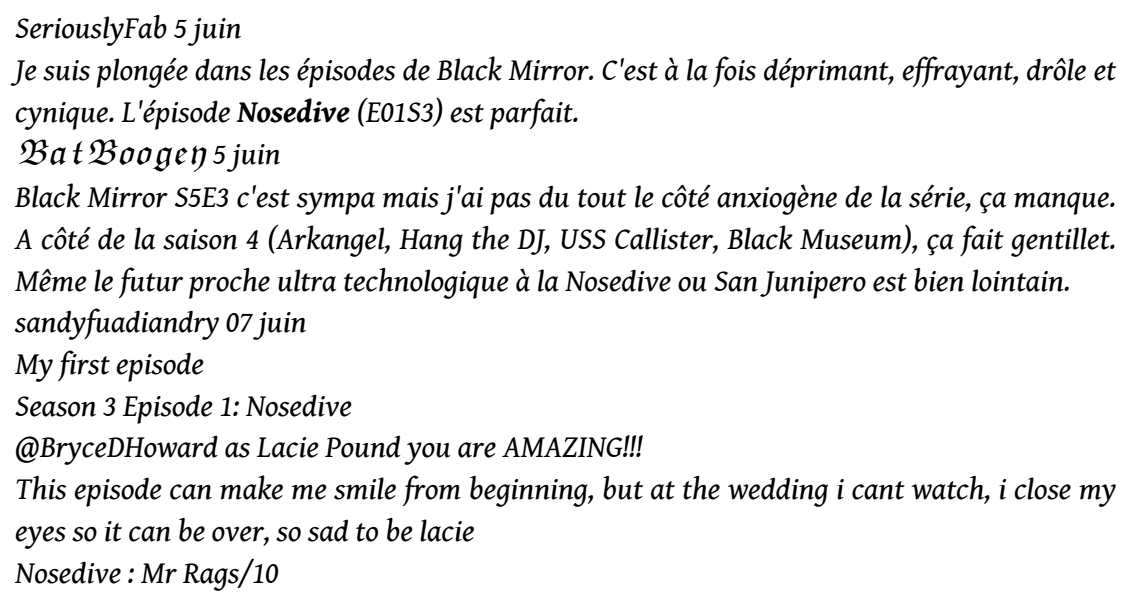

9 En portant un jugement non pas en termes de juste ou de bien mais en relation avec une vie souhaitable et souhaitée, comme le préconise également Iris Murdoch inspirant les propositions de Stanley Cavell ${ }^{18}$, au regard de ces séquences mettant en scène la chute d'une jeune fille dans une société hiérarchisée par un système technique totalitaire de notation qui ne donne pas accès aux mêmes biens (logement, vol en avion, voiture de location) ou sociabilités (amie influenceuse, collègue placardisé), il s'agit de viser par l'énoncé public que constitue un tweet auto-indexé par le mot-clé \#nosedive à une transformation d'un certain usage numérique émergent autour duquel l'épisode de Black Mirror fabule suivant la méthodologie de la «technologie fictive » décrite plus haut.
@Ganeshdeux 5 juin
À chaque épisode on se disait
"Waah je suis content de ne pas vivre dans ce monde "
Cf. 15 millions de mérites, Nosedive, White christmas

10 Le rapprochement avec certains services déjà en vigueur de chauffeurs de voiture, livreurs de pizzas ou de loueurs d'appartements basés sur des applications mobiles, telles que Uber, Delivroo et dans une certaine mesure Arbnb ou Blablacar, est alors rapidement effectué par certain.es qui peuvent ainsi dénoncer l'imperfectionnisme moral du monde connecté contemporain en miroir du monde projeté dans "Chute Libre ".

\section{@RDCresearcher 30 mai}

This is the "Nosedive » episode of \#BlackMirror come to life + about to happen y'all... this is potentially dangerous. \#Uber says it will kick off \#riders for low ratings https://cnn.it/

2QwihVC @Uber how do you plan to detect racism + forms of discrimination w/this system?

11 L'acceptabilité sociale d'un système non plus seulement de recommandations de biens et de services, comme c'est le cas pour des sites tels que Tripadvisor ou Amazon, mais désormais d'individus comme le met en scène "Chute Libre» est problématisée par cette mise en commun des réceptions et des verbalisations. L'imperfectionnisme moral est pointé dans les séquences de mise en calcul des interactions sociales qui abrègent considérablement le temps de communication interpersonnelle. Entre deux gestes d'activation de l'application permettant de quantifier de 1 à 5 étoiles la situation sociale qui vient d'être vécue (commande au salon de thé, rencontres de collègues dans l'ascenseur), le conformisme social consiste essentiellement aux sourires forcés 
auxquels il s'agit de s'entrainer, comme dans la première séquence de l'épisode où l'héroïne face à son miroir se prépare à une journée conforme, c'est à dire ponctuée de phrases gentillettes et de risettes, et mis en conversation dans les commentaires de l'épisode sur le réseau socio-numérique public matérialisé par Twitter. De même, lors des débats auxquels j'ai pu assister les questionnements mettaient en discussion certains aspects jugés problématiques dans la numérisation de la société.

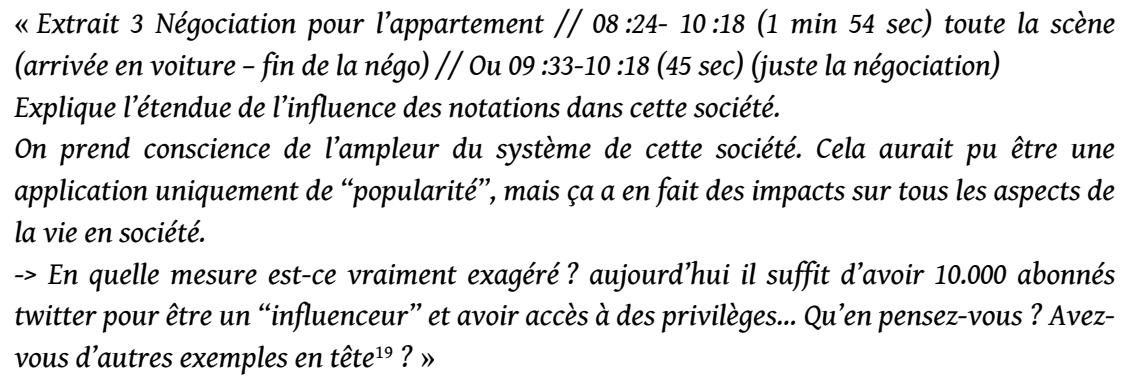

Á l'aune de l'hypothèse du perfectionnisme moral, c'est bien une manière de vivre qui met en avant les relations personnelles et la transformation de soi et de la société qui est mise en question dans l'épisode «Chute Libre » et les mises en débats qu'il inspire. C'est toute la performativité d'un jeu de langage multimodale qui de fait rend possible de ne pas seulement dire le bien et le mal, le juste et le vrai, mais d'enquêter en public sur le monde connecté dans lequel nous voulons vivre ${ }^{20}$. Suivant Sandra Laugier, il se confirme ici que le perfectionnisme moral soit une dimension continue de la vie morale et non le seul exercice de choix explicites, c'est à dire qu'il s'agit de prêter attention à cette texture fine de notre vie de tous les jours au sein de laquelle que notre caractère, se jouent, se forment et se reforment de manière incessante ${ }^{21}$.

\section{2. "Chute Libre" : une voie à soi : quelle désobéissance civile numérique pour sortir du spectacle de la critique ? Expérimenter la voix du contre-faire}

13 Le couplage entre le monde projeté dans "Chute Libre », tramé d'imperfectionnisme moral par la réduction de l'intersubjectivité à l'intercalculabilité, et le perfectionnisme moral que la mise en conversation des réceptions dans la salle ou internet peut entrainer n'épuise cependant pas le tout de l'analyse des corpus assemblés pour cet article.

\subsection{Spectacle de la critique et confusionnisme fictionnel : le cas de la notation sociale en Chine}

Dans les débats auxquels nous avons participé, l'exemple chinois est souvent convoqué comme terrain d'application de la fiction imaginée dans « Chute Libre ».

Extrait 2 - Scène du salon de thé // $03: 02$ - 04 :00 (58 sec)

Montre l'usage de l'application, la manière dont elle change les rapports sociaux

$\rightarrow$ Exemple de la Chine

http://www.journaldugeek.com/2016/10/26/chine-note-confiance-citoyens/

-> Exemple également de Uber, où l'on donne une note à ses chauffeurs, quasiment devant

eux - mais aussi : ils nous attribuent une note (et on ne peut s'empêcher d'aller regarder 
combien on a reçu en moyenne http://www.frandroid.com/comment-faire/tutoriaux/ 312387_tuto-obtenir-note-lapplication-uber) ${ }^{22}$ "notation sociale" se trouvent commentées et auto-indexées par le tag \#nosedive.

@DrSaurowise 28 mai

Avec les récents événements en Chine, j'ai voulu me refaire Nosedive, et c'est vraiment percutant à quel point l'être humain n'est pas fait pour être contenir ses émotions.

@fgascoin 21 mai

Le côté obscur de la technologie ... La saison 5 de \#BlackMirror sort en ce moment et la chine continue de mettre en oeuvre l'épisode 1 de la saison 3 (\#nosedive) \#innovation \#socialnetwok @Being_French 25 mai

Les Chinois tiennent à démontrer que 1984 d'Orwell ou Nosedive dans la série Black Mirror ne sont plus des fictions. Ils y arrivent très bien! L'enfer sur terre nous viendra du Levant!

Le "péril jaune " semble être revisité à l'aune du spectacle de la critique et d'une fictionnalisation d'expérimentations technologiques émergentes comme ce tweet l'énonce sincèrement :

@S_Team_Approved 22 mai

Je ne connais pas la Chine et n'ai pas creusé plus que ça le sujet en référence mais soit ça va vraiment aussi loin et c'est alors plus que terrible ou bien l'occident projette ses craintes les plus fortes sur ce modèle de contrôle social pour la peur? \#nosedive

De fait, ces expériences dites de «notation sociale » se trouvent à leur tour fictionnées en miroir de la méthode de la «technologie fictive» de Black Mirror. Les chercheur.es en la matière rappelle que, certes, il existe des projets de calcul du

score du consommateur utilisé pour classer les citoyens sur la base d'une variété de facteurs, comme la loyauté envers le gouvernement chinois et la fidélité aux marques chinoises, à partir de ses interactions sur les médias sociaux et des achats effectués en ligne. Avoir un score élevé permet un accès plus facile à des prêts, simplifie l'accès à l'emploi et donne priorité lors de démarches administratives. Un faible score, ou le fait d'être associé à quelqu'un avec un score faible peut avoir une série de conséquences négatives : la baisse de la vitesse de l'internet, l'accès plus difficile à des offres d'emploi, des prêts ou des démarches administratives ${ }^{23}$.

Sesame Credit qui propose ce système d'évaluation du crédit social individuel est développé par Ant Financial Services Group, filiale du groupe chinois Alibaba et associé du gouvernement chinois. Il faut, pour parfaire la réalité empirique du « crédit social » à la chinoise, remettre ce système dans un plan gouvernemental plus global qui cherche à pallier au manque de confiance à l'intérieur même du pays pour le made in china notamment en ce qui concerne les problèmes de sécurité alimentaire, de fraude, et les marchandises de contrefaçon. Il s'agit, par ce dispositif des technologies de contrôle associant reconnaissance faciale, traitement de données et notation sociale de promouvoir l'intégrité et la crédibilité au sein de la société chinoise elle-même.

Dans ce cas, la technologie fictive réalisée dans l'épisode «Chute Libre » est reçue, dans un contexte social loin de Pékin, dans les commentaires et les débats, comme la réalisation d'une société connectée d'intercalculabilité à propos de laquelle, faute d'y interagir au quotidien, sont proférées des jugements moraux normatifs qui pourraient, par ailleurs, être bien fondés et se trouvent dénoncés par les citoyens dissidents.

Le perfectionnisme moral, comme pratique de transformation du monde par le jugement par soi et avec les autres dans le milieu de la vie quotidienne, se trouve empêché dans ce contexte lointain et la fausse éthique du bien et du mal, reproduisant la pratique de l'étiquetage des entrepreneurs de morale ${ }^{24}$, demeure le seul ressort de 
citoyens du monde rendus impuissants de par leur posture de spectateurs lointains jugeant sans savoir. Se pose alors la question du comment sortir de la critique du spectacle pour réactiver un perfectionnisme moral et sa vertu transformatrice?

\subsection{Se déconnecter et parler vrai : perfectionnisme moral, le retour}

21 L'épisode "Chute libre » présente, au sein de la série, la particularité d'un dénouement ouvert sur le possible de la déconnexion, de la sortie du système de notation. Les deux séquences mettant en scène cette voie de sortie sont les plus bavardes de l'épisode, hormis les séquences de longue discussion ente la personnage principale et son frère, outsider critique du système qui l'embarque dans une course à l'influence pernicieuse.

La première d'entre elles met en présence une camionneuse bienveillante qui prend en stop l'héroïne, qui faute de note sociale suffisante, pour les entrepreneurs de sa cité du calcul n'a pu prendre l'avion et espère toujours se rendre au mariage de sa meilleure amie, influenceuse par ailleurs. Cette femme, chargée d'histoires personnelles, raconte l'histoire de sa défection de l'économie de l'influence. En effet, bien que possédant un score social lui permettant des accès aux soins, elle perdra son mari d'un cancer, qui lui n'a rien de numérique.

Son choix individuel, voire solitaire, de la défection (exit) plutôt que de la protestation (voice), pour reprendre les termes d'Albert 0 . Hirschman ${ }^{25}$, introduit pour l'héroïne une modulation nouvelle, celle de la "voix à soi » au double sens d'écoute de soi avec les autres avec lesquels devenir des êtres perfectibles.

Cette défection critique ou néo-luddite, aménageant un début d'autonomie de vie dans un véhicule nomade et hospitalier vis-à-vis des outsiders, pourrait représenter la voix de sortie d'une société stratifiée, inégalitaire et injuste.

La dernière partie de "Chute Libre" peut être qualifiée de jubilatoire avec une séquence finale plus tant bavarde, performant que c'est par la conversation avec autrui que l'on peut s'accorder sur une modalité de bonne vie au sens d'un jugement moral intersubjectif, mais encore plus authentiquement transgressive du conformisme de l'évitement par l'adoption d'un "parler vrai». Phrases et mots libérateurs sont échangés par deux prisonniers dont l'héroïne; insultes, noms d'oiseaux et autres propos absurdes ponctuent cette dernière scène de "Chute libre" sonorisant la fameuse agonistique du langage mise en lumière par Michel Foucault qui insiste sur le caractère de joute oratoire des jeux des langages, la parrhêsia pouvant renvoyer à « la manière dont le sujet fait l'expérience de lui-même dans un jeu de vérité où il a rapport à soi $^{26} »$.

Retrouver une voix là où régnait la notification sonore. Faire sonner sa verve là où la gamme des chiffres étaient réduites à 5 étoiles, c'est encore actualiser un perfectionnisme moral qui « engage et aide chacun à se faire confiance, et à trouver sa voix », et cela " contre la morale des devoirs abstraits, des faux choix rationnels et des calculs d'utilitéé ${ }^{27}$."

27 Cette dernière scène est une bataille de mots entre deux outsiders qui jouent le conflit là où elle et ils ont imité de façon quasi mutique le conformisme du plus petit dénominateur social. Elle met en représentation in fine un acte de désobéissance civile par la performativité du dissensus, par le refus d'un court-circuit algorithmique des conversations, par la réappropriation d'une voix propre qui ne compte plus en notre 
nom. Á la manière d'Henry David Thoreau et de Ralph Waldo Emerson, dont «la position est simple dans son principe : on a non seulement le droit mais le devoir de résister, et donc de désobéir, lorsque le gouvernement agit contre ses propres principes. Thoreau refuse de reconnaitre le gouvernement comme sien, et refuse de lui donner sa voix, refuse qu'il parle en son nom, lorsque par exemple il promeut l'esclavage ou fait la guerre au Mexique. C'est là un sentiment politique très fondamental, qu'on retrouvait récemment dans les oppositions internes à la guerre en Irak : Not in our name ${ }^{28}$.»

\section{Conclusion : La voix du contre-faire}

En conclusion, je voudrais plaider pour une autre forme de désobéissance civile qui ne passe par uniquement par le parler vrai et la voix retrouvée mais également par la possibilité de fabriquer un autre monde connecté, ce que je désigne par le «contrefaire ${ }^{29}$ ». Dans le cadre des espaces tiers de la civilité technologique nommés fablab ou hackerspace et de par la mobilisation d'acteurs autour du chantier de la fabrication personnelle initié par le mouvement des makers, des fablabs et acteur.es des low tech, il est encore possible de réinitialiser au quotidien d'autres scénarii socio-techniques afin de transformer le monde connecté pour soi et les autres suivant le jugement d'une vie connectée bonne à vivre et soutenable au plan d'une écologie commune pour les humains et les non-humains.

\section{BIBLIOGRAPHIE}

AKRICH, Madeleine, LATOUR, Bruno, CALLON, Michel, éds., Sociologie de la traduction : textes fondateurs, Paris, Presses des Mines, 2006.

ALLARD, Laurence, «L'engagement du chercheur à l'heure de la fabrication personnelle », Hermès, 2015/3, n 73, p. 159-167, https://www.cairn.info/revue-hermes-la-revue-2015-3-page-159.htm.

ALLARD, Laurence, « Dire et voir la fiction. \#doublescreening \#blackmirror », in D'un écran, l'autre, éd. Jean CHÂTEAUVERT et Gilles DelAVAUd, Paris, INA-L'Harmattan, coll. « Les médias en actes », 2016, p. 501-513.

ALLARD, Laurence, « Dans quel monde voulons-nous être connectés? Transhumanisme vs companionism ", Nectart, 2016/2, n 3, pp. 125-132, https://www.cairn.info/revuenectart-2016-2-page-125.htm.

BECKER, Howard, Outsiders. Études de sociologie de la déviance [1 $1^{\mathrm{e}}$ éd. : 1963], préface de Jean-Michel CHAPOULIE, trad. franç. de Jean-Pierre BRIAND et Jean-Michel CHAPOULIE, Paris, Métaillé, 2012. BROOKER, Charlie, JONES, Annabel, with ARNOPP Jason, Inside Black Mirror, New York, Crown Archetype, 2018. 
CAMPION, Benjamin, « Festival Séries Mania : conférence sur Black Mirror le 15 avril », site de Libération, 14 avril 2017, http://feuilletons.blogs.liberation.fr/2017/04/14/festival-series-maniaconference-sur-black-mirror-le-15-avril/.

CAVELL, Stanley, Conditions nobles et ignobles. La constitution du perfectionnisme émersonien [ $1^{\mathrm{e}}$ éd. : 1990], trad. franç. par Christian FOURNIER et Sandra LAUGIER, Paris, Editions de l'éclat, coll. « Tiré à part », 1993.

CAVELL, Stanley, La projection du monde. Réflexions sur l'ontologie du cinéma [1 $1^{\mathrm{e}}$ éd. : 1971], Paris, Belin, 1999.

CAVELL, Stanley, Dire et vouloir dire. Livre d'essais [ $1^{\mathrm{e}}$ éd. : 1969], trad. franç. par Sandra LAUGIER et Christian FOURNIER Paris, Cerf, coll. « Passages », 2009.

CORCUfF, Philippe, Polars, philosophie et critique sociale, dessins de CHARB, Paris, Textuel, coll. « Petite encyclopédie critique », 2013.

DEWEY, John, Le public et ses problèmes [ $1^{\mathrm{e}}$ éd. : 1927], trad. franç. et préface de Joëlle ZASK, PauTours-Paris, Publications de l'Université de Pau-Farrago-Léo Scheer, 2003.

FORESTIER, Florian, « Émergence de nouvelles formes de contrôle social en Chine », site de Futuribles, « Note de veille », 11 octobre 2018, https://www.futuribles.com/fr/article/emergencede-nouvelles-formes-de-controle-social-e/.

Foucault, Michel, « Foucault » [1 $1^{\mathrm{e}}$ éd. : 1984], in Dits et écrits II, 1976-1988, Paris, Gallimard, coll. "Quarto », édition établie sous la direction de Daniel DEFERT et François EWALD, n 345, 2001, p. 1450-1455.

GROS, Frédéric, «La parrhêsia chez Foucault (1982-1984) », in Foucault. Le courage de la vérité, éd. Frédéric GROS, Paris, PUF, coll. « Débats philosophiques », 2002, p. 155-165.

HIRSCHMAN, Albert O., Exit, voice, loyalty. Défection et prise de parole [1 $1^{\mathrm{e}}$ éd. : 1970], préface de Pascal DELWIT, trad. franç. par Claude BESSEYRIAS, Bruxelles, Éditions de l'Université de Bruxelles, 2017.

LAUGIER, Sandra, «Le modèle américain de la désobéissance civile, de Thoreau à nos jours ", Multitudes, $\mathrm{n}^{\circ} 24$ [online], printemps 2006, https://www.multitudes.net/wp-content/uploads/ 2006/04/24-laugier.pdf.

LAUGIER, Sandra, éd., La voix et la vertu. Variétés du perfectionnisme moral, Paris, PUF, coll. « Éthique et philosophie morale », 2010.

LAUGIER, Sandra, «Présentation. L'autre voie de la philosophie morale », in La voix et la vertu. Variétés du perfectionnisme moral, éd. Sandra LAUGIER, Paris, PUF, coll. «Éthique et philosophie morale », 2010, p. 1-30.

LORENZINI, Daniele, Éthique et politique de soi : Foucault, Hadot, Cavell et les techniques de l'ordinaire, Paris, Vrin, coll. «Problèmes \& controverses », 2015.

MERJAGNAN, Norbert, « Black Mirror : "La SF cauchemarde le monde parce qu'elle sait que le bonheur est possible" », entretien avec Guillaume LEDIT, Usbek \& Rica, 11/01/2018, https:// usbeketrica.com/article/black-mirror-sf-cauchemarde-dystopie-merjagnan.

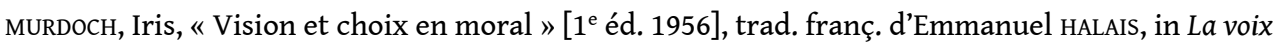
et la vertu. Variétés du perfectionnisme moral, éd. Sandra LAUGIER, Paris, PUF, coll. « Éthique et philosophie morale », 2010, p. 61-88. 


\section{NOTES}

1. Norbert Merjagnan, «Black Mirror : "La SF cauchemarde le monde parce qu'elle sait que le bonheur est possible" », entretien avec Guillaume Ledit, Usbek \& Rica, 11/01/2018, https:// usbeketrica.com/article/black-mirror-sf-cauchemarde-dystopie-merjagnan.

2. Voir Stanley Cavell, Dire et vouloir dire. Livre d'essais [1 $1^{\mathrm{e}}$ éd. : 1969], Paris, Cerf, coll. « Passages », 2009.

3. Voir Stanley Cavell, La projection du monde. Réflexions sur l'ontologie du cinéma [1 ${ }^{\mathrm{e}}$ éd. : 1971], Paris, Belin, 1999.

4. Stanley Cavell, Conditions nobles et ignobles. La constitution du perfectionnisme émersonien [ $1^{\mathrm{e}}$ éd. : 1990], Paris, Editions de l'éclat, coll. « Tiré à part », 1993, p. 50.

5. «Chute libre » a été diffusé pour la première fois le 21 octobre 2016 sur Netflix. L'histoire se déroule dans un monde où chaque personne note les autres de 0 à 5 , les mieux notés ayant accès à de meilleurs services. Lacie ne vit que pour améliorer sa note de 4,2 et cherche à quitter l'appartement qu'elle partage avec son frère, qui méprise le système de notation, pour les beaux quartiers. Elle se montre donc extrêmement polie et recherche toujours à obtenir l'approbation générale, quitte à mal noter un collègue qu'elle apprécie. Lacie est également en admiration devant Naomi, sa belle amie d'enfance qui a toujours maintenu sa note au-dessus de 4,6 dont elle garde le souvenir en scrutant ses profils sur les réseaux sociaux et en conservant une peluche qu'elles ont faite ensemble alors qu'elles avaient 5 ans. Lacie trouve l'appartement idéal où elle pourrait vivre une vie de couple lisse et idéale mais elle doit obtenir la note minimum de 4,5 pour avoir droit à une réduction de loyer. Sur l'avis d'un conseiller en cotation, elle tente de dresser une image plus personnelle d'elle-même et poste une photo de sa peluche. Peu après, Naomi la contacte : elle vient de se fiancer et demande à son amie d'enfance d'être sa demoiselle d'honneur. Lacie y voit une chance unique, par un discours millimétré, de faire grimper sa note et prépare alors minutieusement son texte. (Source : Wikipédia)

6. Laurence Allard, « Dire et voir la fiction. \#doublescreening \#blackmirror », in D'un écran, l'autre, éd. Jean Châteauvert et Gilles Delavaud, Paris, INA-L'Harmattan, coll. «Les médias en actes », 2016, p. 501-513.

7. Le fil de tweets correspondant au mot-dièse \#nosedive https://twitter.com/search? $\mathrm{q}=$ nosedive \&src=typd .

8. Allard, « Dire et voir la fiction. \#doublescreening \#blackmirror », op. cit.

9. Charlie Brooker, Annabel Jones, with Jason Arnopp, Inside Black Mirror, New York, Crown Archetype, 2018.

10. Madeleine Akrich, Bruno Latour, Michel Callon, éds., Sociologie de la traduction: textes fondateurs, Paris, Presses des Mines, 2006.

11. Voir Philippe Corcuff, Polars, philosophie et critique sociale, dessins de Charb, Paris, Textuel, coll. «Petite encyclopédie critique », 2013.

12. Cf son dernier roman, Les furtifs, Clamart, La Volte, 2019 présenté ainsi sur le site de l'éditeur : «Les Furtifs vous plonge dans un futur proche et fluide où le technococon a affiné ses prises sur nos existences. Une bague interface nos rapports au monde en offrant à chaque individu son alter ego numérique, sous forme d'IA personnalisée, où viennent se concentrer nos besoins vampirisés d'écoute et d'échanges. Partout où cela s'avérait rentable, les villes ont été rachetées par des multinationales pour être gérées en zones standard, premium et privilège selon le forfait citoyen dont vous vous acquittez. La bague au doigt, vous êtes tout à fait libres et parfaitement tracés, soumis au régime d'auto-aliénation consentant propre au raffinement du capitalisme cognitif. », https://lavolte.net/livres/les-furtifs-alain-damasio/.

13. Cf par exemple le descriptif de la conférence sur Black Mirror le 15 avril 2017 lors du Festival Séries Mania : «Quand Black Mirror révèle les dérives de nos sociétés. Pour son arrivée sur Netflix, Black Mirror a fait fort. Chute libre (S03E01) présente un monde où chaque individu est noté par ses 
semblables. Résultat : femmes et hommes ne sont plus libres et égaux en droit mais hiérarchisés "en like". Effrayant non? Mais qu'on se rassure, ce n'est que de la fiction. Sauf que... Depuis, la Chine envisage de classer ses citoyens en fonction de leurs données personnelles. Aux USA, prière de déclarer votre compte Facebook pour passer la douane. Et en France, le "méga-fichier" TES cristallise les tensions. Alors quoi ? Ce "miroir noir" serait en fait plus prophétique que dystopique ? Laissons nos peurs de côté et ouvrons le débat avec nos spécialistes. Révisez-bien votre épisode avant la séance! ", in Benjamin Campion, «Festival Séries Mania : conférence sur Black Mirror le 15 avril », site de Libération, 14 avril 2017, http://feuilletons.blogs.liberation.fr/ 2017/04/14/festival-series-mania-conference-sur-black-mirror-le-15-avril/.

14. Voir Cavell, La projection du monde, op. cit.

15. La théorie de l'enquête chez John Dewey est indissociable de sa conception vitaliste de l'expérience comme écologie de l'expérimentation. En partant d'une situation indéterminée, elle consiste à mettre en place un processus expérimental permettant de parvenir à une situation unifiée et ce par la constitution d'un public attaché à enquêter pour solutionner un problème dans une perspective de méliorisme. Cf John Dewey, Le public et ses problèmes [1 $1^{\mathrm{e}}$ éd. : 1927], PauTours-Paris, Publications de l'Université de Pau-Farrago-Léo Scheer, 2003.

16. Allard, « Dire et voir la fiction. \#doublescreening \#blackmirror », op. cit.

17. Ibid.

18. Dans «Vision et choix en morale» [ 1 e éd.: 1956], Iris Murdoch rappelle que dans la philosophie morale dominante «un concept moral est une définition objective d'un certain domaine d'activité, plus une recommandation ou une interdiction ", repris in La voix et la vertu. Variétés du perfectionnisme moral, éd. Sandra Laugier, Paris, PUF, coll. «Éthique et philosophie morale », 2010, p. 65.

19. Exemple de fil conducteur de débats, Forum des Images, mai 2017.

20. Allard, « Dire et voir la fiction. \#doublescreening \#blackmirror », op. cit.

21. Sandra Laugier, «Présentation. L'autre voie de la philosophie morale ", in La voix et la vertu. Variétés du perfectionnisme moral, op. cit., p. 1-30.

22. Exemple de questions posées lors du débat au Festival Séries Mania, 15 avril 2017.

23. Florian Forestier, "Émergence de nouvelles formes de contrôle social en Chine ", site de Futuribles, « Note de veille », 11 octobre 2018, https://www.futuribles.com/fr/article/emergencede-nouvelles-formes-de-controle-social-e/.

24. Howard Becker, Outsiders. Études de sociologie de la déviance [1 $1^{\mathrm{e}}$ éd. : 1963], Paris, Métaillé, 2012.

25. Albert O. Hirschman, Exit, voice, loyalty. Défection et prise de parole [1 e éd.: 1970], Bruxelles, Éditions de l'Université de Bruxelles, 2017.

26. Selon les termes de Michel Foucault pour caractériser la « subjectivité » dans « Foucault » $\left[1^{\mathrm{e}}\right.$ éd. : 1984], in Dits et écrits II, 1976-1988, Paris, Gallimard, coll. « Quarto », 2001, p. 1452 ; sur l'usage foucaldien de la notion de parrhêsia puisée dans la Grèce antique, voir Frédéric Gros, « La parrhêsia chez Foucault (1982-1984) », in Foucault. Le courage de la vérité, éd. Frédéric Gros, Paris, PUF, coll. « Débats philosophiques », 2002, p. 155-165.

27. Quatrième de couverture de La voix et la vertu. Variétés du perfectionnisme moral, éd. Sandra Laugier, op. cit.

28. Sandra Laugier, "Le modèle américain de la désobéissance civile, de Thoreau à nos jours ", Multitudes, $\mathrm{n}^{\circ} 24$ [online], printemps 2006, https://www.multitudes.net/wp-content/uploads/ 2006/04/24-laugier.pdf.

29. Laurence Allard, "L'engagement du chercheur à l'heure de la fabrication personnelle ", Hermès, 2015/3, $\mathrm{n}^{\circ}$ 73, p. 159-167, https://www.cairn.info/revue-hermes-la-revue-2015-3page-159.htm. 


\section{RÉSUMÉS}

Cet article voudrait s'attarder sur un épisode bien connu la série britannique Black Mirror, créée Charlie Brooker et diffusée sur Channel 4 (2011-2014), puis sur Netflix (2016-), « Nosedive » (ou "Chute libre»), premier épisode de la troisième saison (2016). Nous nous attacherons en particulier à typifier les formes de vie socio-numériques qui y sont caricaturées suivant une prospective d'imperfectionnisme moral. Cet horizon d'imperfectionnisme moral vient questionner la vision de Stanley Cavell de constituer les relations interpersonnelles comme substrat nécessaire à la possibilité de transformation de notre société. Nous proposerons une voie/voix critique permettant échapper à une passive consommation de la dystopie au profit d'un « contre-faire » et d'une re-fabrication d'un autre monde connecté possible écologiquement soutenable.

This article would like to focus on a well-known episode of the British series Black Mirror, created by Charlie Brooker and broadcast on Channel 4 (2011-2014), then on Netflix (2016-), "Nosedive", the first episode of the third season (2016). We will focus in particular on typifying the socialdigital forms of life that are caricatured in it according to a prospective of moral imperfectionism. This horizon of moral imperfectionism questions Stanley Cavell's vision of interpersonal relations as a necessary substratum for the possibility of transforming our society. We will propose a critical path/voice that allows us to escape the passive consumption of dystopia in favor of a "contre-faire" and a re-creation of another sustainable connected world.

\section{INDEX}

Mots-clés : Black Mirror, imperfectionnisme, dystopie, low tech, vie connectée

Keywords : Black Mirror, imperfectionism, dystopia, low tech, connected

\section{AUTEUR}

\section{LAURENCE ALLARD}

Laurence Allard est maîtresse de conférences en sciences de l'information et de la communication à l'Université de Lille et membre de l'IRCAV (Institut de recherche sur le cinéma et l'audiovisuel, Université Sorbonne Nouvelle, EA 185). Elle étudie les usages citoyens du numérique et s'est spécialisée dans la culture mobile. Elle a co-fondé l'association Labo Citoyen engagée dans les dispositifs low tech de captation environnementale. Son site professionnel: https://culturesexpressives.fr/doku.php.

Laurence Allard is reader in Information and Communication Sciences in Lille University and member of IRCAV (Institute for Cinema and Audiovisual Research, Sorbonne Nouvelle University, EA 185). She studies the citizen uses of digital technology and specializes in mobile culture. She co-founded the association Labo Citoyen, which is involved in low tech environmental capture devices. Her professional website: https://culturesexpressives.fr/doku.php. 